\title{
La dinámica del empleo en la frontera norte de Uruguay Fernando Correa Alsina*
}

\begin{abstract}
Resumen
Uruguay comparte una extensa frontera con Brasil. En esta zona, los habitantes de ambas márgenes realizan sus compras en aquel país que coyunturalmente resultan más convenientes. Las fuertes alteraciones que ha sufrido el tipo de cambio real bilateral han determinado que la demanda se haya trasladado a un lado y otro de la frontera de forma alternada, repercutiendo en el nivel de empleo generado en dos departamentos fronterizos de Uruguay. En este artículo se presenta un modelo teórico que intenta recoger los principales elementos del fenómeno analizado. Las estimaciones econométricas realizadas validan el modelo planteado y muestran que los mercados laborales de ambos lados de la frontera no están lo suficientemente integrados como para amortiguar los impactos derivados de las variaciones de la demanda.
\end{abstract}

Palabras clave: frontera, consumo, precios, empleo, integración.

\begin{abstract}
Uruguay shares a long border with Brazil. There, inhabitants from both sides of the border shop in whichever country they find the cheapest prices. As a consequence of severe changes in the bilateral real exchange rate, the demand moves alternately from side to the other of the border, affecting the level of employment in two Uruguayan border provinces. The theoretical model outlined in this article intends to capture the main elements of the analyzed situation. The econometric estimates carried out validate the stated model and show that the labor markets on both sides of the border are not integrated enough to smooth the impact of demand movements.
\end{abstract}

Keywords: border, consumption, prices, employment, integration.

* Universidad de la República, Regional Norte-Salto, Uruguay. Correo electrónico: saltoriental@hotmail.com 


\section{Introducción}

Uruguay comparte al noreste una extensa frontera con el Brasil (figura 1). Próximo a la misma habita un porcentaje significativo de la población de los departamentos de Artigas $(79,2 \%)$ y Rivera $(73,4 \%)$. En particular, se ubican las dos capitales departamentales, las que se encuentran muy cercanas a otras dos ciudades brasileñas: Quaraí y Santana do Livramento.

La población uruguaya de esta zona, al igual que la brasileña, acostumbra realizar sus compras de bienes de consumo en aquel lado de la frontera que se encuentra circunstancialmente más barato. La gran variabilidad exhibida en el pasado por el tipo de cambio real bilateral entre

\section{Figura 1}

\section{Frontera Uruguay-Brasil}

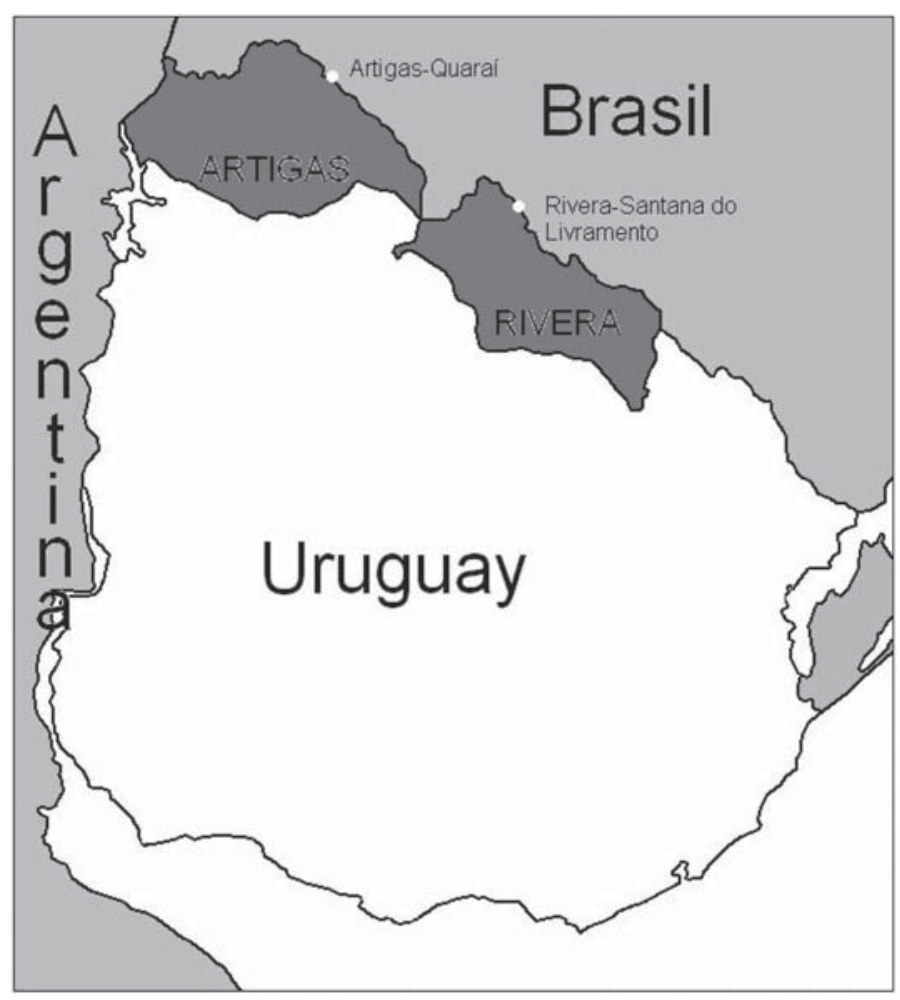


ambos países ha determinado que el consumo se traslade a un lado y otro de forma alternada.

El fenómeno del consumo transfronterizo comprende a la mayoría de los bienes y también, aunque en menor medida, a los servicios. Los principales bienes consumidos en el país vecino son alimentos, bebidas, cigarrillos, vestimenta, muebles, electrodomésticos, combustibles y gas. Asimismo, corresponde destacar que esta costumbre no abarca solamente a la población de las ciudades fronterizas sino también a aquellos poblados cercanos a la frontera. En este sentido, debe tenerse presente que aproximadamente 5\% de la población del Uruguay vive a menos de 10 kilómetros de distancia, por vía terrestre, de una ciudad brasileña.

La posibilidad que tienen los consumidores fronterizos de elegir el lugar de compra les ha permitido sacar un importante provecho en términos de reducción de su costo de vida. A su vez, se han visto afectados de forma significativa cuando el gobierno ha implementado medidas más estrictas de control aduanero, llegando incluso a las denominadas políticas de "cero kilo". No obstante, cabe señalar que las mismas han sido esporádicas y por periodos relativamente cortos.

Así como el fenómeno del consumo transfronterizo tiene su lado favorable para los habitantes de estos departamentos, también tiene su lado negativo. En la medida en que la demanda de bienes y de servicios se traslada a un lado y otro de la frontera, la generación de empleo se vuelve más inestable que en un departamento del interior del país. En este sentido, debe destacarse que no solamente se ve afectada la generación de empleo en los sectores que producen los bienes y servicios ya mencionados, sino también y fundamentalmente en el sector del comercio minorista.

El objetivo de este trabajo es determinar en qué medida el fenómeno del consumo transfronterizo afecta la tasa de empleo de la población uruguaya de esta zona. Si los mercados laborales de ambas márgenes de la frontera estuvieran bien integrados, las relocalizaciones del consumo de un país a otro darían lugar a relocalizaciones del empleo, sin afectar las tasas de ocupación de la población que habita en ambas márgenes. Por el contrario, si los mercados laborales no están lo suficientemente integrados, habrá repercusiones directas sobre los mismos.

Los resultados aquí presentados muestran que el nivel de empleo en la frontera está directamente correlacionado con el tipo de cambio real bi- 
lateral entre Uruguay y Brasil. Esto pone de manifiesto que los mercados laborales no están lo suficientemente integrados como para amortiguar las fluctuaciones derivadas del fenómeno de las relocalizaciones del consumo entre ambas márgenes.

El trabajo se organiza en cinco secciones: en la primera se reseñan los principales estudios realizados relativos a la temática analizada; en segundo lugar, se presenta un modelo simplificado que recoge los principales elementos del fenómeno aludido; tercero, se realiza un análisis gráfico de la relación entre las variables relevantes del modelo; cuarto, se realizan estimaciones econométricas para validar el modelo sugerido; finalmente, se resumen las principales conclusiones y derivaciones del estudio.

\section{Antecedentes sobre el tema}

El fenómeno del consumo transfronterizo ha sido analizado desde diferentes perspectivas en la literatura económica. Existe un conjunto de investigaciones que se ha orientado a determinar las causas del mismo, focalizándose en explicar por qué existen las diferencias de precios entre países. Un estudio relevante en este sentido (Engel y Rogers, 1996) examinó los precios relativos al consumo entre ciudades de Estados Unidos y Canadá, encontrando que ambos mercados están segmentados en una medida que no puede ser explicada por la distancia, las restricciones formales al comercio y la rigidez de los precios. Esto sugiere la existencia de otras causas, tales como la segmentación de los mercados de insumos y laborales. Por el contrario, otra investigación realizada entre países no limítrofes (Parley y Wei, 2001) encuentra que una parte sustancial de la segmentación internacional de mercados se puede explicar conjuntamente por la distancia, los costos unitarios de transporte y la variabilidad del tipo de cambio.

El fenómeno de las diferencias de precios entre países también ha sido analizado desde la perspectiva de su impacto en el costo de vida de los habitantes fronterizos. En particular para el caso de Uruguay, las estimaciones realizadas (Correa, 2006:51) muestran que durante el periodo 1995-2005 el costo de vida en alimentos y bebidas en la frontera fue en promedio $39.4 \%$ inferior al correspondiente al interior del país. Esta diferencia de costo de vida experimentó fuertes fluctuaciones básicamente determinadas por la inestabilidad del tipo de cambio real bilateral con Brasil. 
Otras investigaciones se han orientado a examinar el impacto que tiene el fenómeno del consumo transfronterizo sobre el nivel de actividad económica a ambos lados de la frontera. Un estudio (Campbell y Lapham, 2004) realizado sobre condados de la frontera Estados Unidos-Canadá analiza la respuesta que tienen el número de establecimientos y su tamaño promedio frente a las fluctuaciones del tipo de cambio real bilateral. Los resultados muestran que el número de establecimientos reacciona rápidamente, mientras que su tamaño es menos sensible a las variaciones de los precios relativos. El impacto sobre el empleo solamente ha sido investigado en episodios puntuales caracterizados por fuertes devaluaciones cambiarias (Harrell y Fischer, 1989). El análisis presentado en el presente trabajo sigue esta misma línea de investigación, focalizándose en el impacto sobre el empleo, aunque con una perspectiva de más largo plazo.

\section{El modelo}

Este modelo representa, en equilibrio parcial, la actividad económica en la frontera entre dos países, por ejemplo, Brasil y Uruguay. A cada lado de la línea fronteriza existen ciudades vecinas, y en cada una de ellas se produce un bien diferenciado que pueden comprar los habitantes de ambas márgenes.

El consumidor determina la cantidad consumida de cada bien de forma de maximizar su nivel de utilidad, tomando como dados los precios de los bienes y de su ingreso, ambos considerados exógenos en este modelo. Su nivel de utilidad $(U)$ se representa adecuadamente por una función del tipo CES:

$$
U\left(x_{u}, x_{b}\right)=x_{u}^{\rho}+x_{b}^{\rho} \quad 0<\rho<1
$$

Donde $x_{u}$ es la cantidad consumida del bien uruguayo y $x_{b}$ es la cantidad consumida del bien brasileño. Los costos de transporte asociados a la compra del bien extranjero son insignificantes y la información relativa al mismo, así como su accesibilidad, es similar a la correspondiente al bien nacional.

Si el consumidor dispone de un ingreso y para gastar, la demanda individual por el bien uruguayo será igual a: 


$$
x_{u}=\frac{y}{p_{u}} \frac{1}{1+\left(p_{b} / p_{u}\right)^{\frac{\rho}{\rho-1}}}
$$

donde $p_{u}$ y $p_{b}$ son los precios de ambos bienes. A su vez, la demanda por el bien de origen brasileño tendrá una expresión simétrica a la anterior.

Por simplicidad, supondremos que en ambas ciudades existe la misma cantidad de consumidores $(P)$, con idéntico ingreso y comportamiento. De esta forma, el nivel de demanda por el bien uruguayo será un múltiplo de la población total.

En lo que respecta a la oferta, consideremos que los dos bienes son producidos utilizando únicamente el factor trabajo con una productividad constante $(r)$. Los trabajadores solamente pueden trabajar en el país donde habitan, existiendo barreras a la entrada en el mercado laboral vecino.

El nivel de producción, y por tanto de empleo, estará determinado por la demanda. De esta manera, igualando oferta y demanda, determinamos el nivel de empleo $\left(E_{u}\right)$ en la margen uruguaya:

$$
\phi E_{u}=2 P \frac{y}{p_{u}} \frac{1}{1+\left(\frac{p_{b}}{p_{u}}\right)^{\frac{\rho}{\rho-1}}}
$$

Finalmente, realizando las trasposiciones necesarias, es posible derivar la tasa de empleo en la ciudad fronteriza de este país:

$$
\frac{E_{u}}{P}=\frac{2}{\phi} \frac{y}{p_{u}} \frac{1}{1+\left(\frac{p_{b}}{p_{u}}\right)^{\frac{\rho}{\rho-1}}}
$$

En resumen, la tasa de empleo resulta determinada, básicamente, por el nivel de ingreso real de la población $\left(\frac{y}{p_{u}}\right)$ y por el tipo de cambio real entre los dos países $\left(\frac{p_{b}}{p_{u}}\right)$. 
A efectos comparativos, consideremos ahora la situación de una ciudad ubicada en el interior del país, lejos de la frontera, con una población total igual a la suma de las dos ciudades fronterizas anteriormente consideradas. Su consumidor típico, a diferencia del anterior, solamente puede adquirir el bien nacional. La distancia y los costos de transporte asociados hacen inaccesible el bien extranjero.

De esta manera, realizando los mismos supuestos que en el caso anterior, la demanda por el bien nacional dependerá únicamente del ingreso real. La producción y por tanto el empleo quedarán determinados básicamente por dos variables: el ingreso real y el tamaño de la población. La tasa de empleo resultará igual a:

$$
\frac{E_{u}}{P}=\frac{2}{\phi} \frac{y}{p_{u}}
$$

De la comparación de las ecuaciones 4 y 5 se desprende que el nivel de empleo en la zona fronteriza se apartará del registrado en el resto del país y que dicho distanciamiento estará determinado por la evolución del tipo de cambio real.

Finalmente, cabe señalar que en este modelo se supuso por simplicidad que el nivel de ingreso de todos los consumidores es idéntico y, por tanto, independiente de su situación de empleo. Este supuesto, poco realista, puede ser relajado asumiendo que el ingreso de un desocupado es equivalente a solamente una porción del ingreso del trabajador ocupado, sin que se alteren los principales resultados del modelo.

\section{Análisis de las variables}

A los efectos de disponer de una primera visión sobre si el modelo presentado anteriormente se ajusta a la realidad del fenómeno objeto de estudio, en esta sección se realiza un análisis gráfico de las series relevantes para el periodo 1990-2009.

En la figura 2 se presentan datos anuales de dos series estadísticas: por un lado, del cociente entre la tasa de empleo en los dos departamentos fronterizos -Artigas y Rivera- tomados en conjunto y la correspondiente a 
Montevideo; por otro, de la evolución del tipo de cambio real bilateral de Uruguay con Brasil. ${ }^{1}$

La primera serie mide el distanciamiento que tiene el empleo en la frontera respecto al interior del país. Corresponde señalar que se eligió la tasa de empleo de Montevideo para representar al interior del país por ser éste un departamento que se ubica muy alejado de la frontera y que a su vez es el de mayor población del Uruguay. La segunda variable mide la evolución de los precios relativos entre los dos países.

Figura 2

Tasa de empleo en la frontera / tasa de empleo en Montevideo y tipo de cambio real Uruguay-Brasil

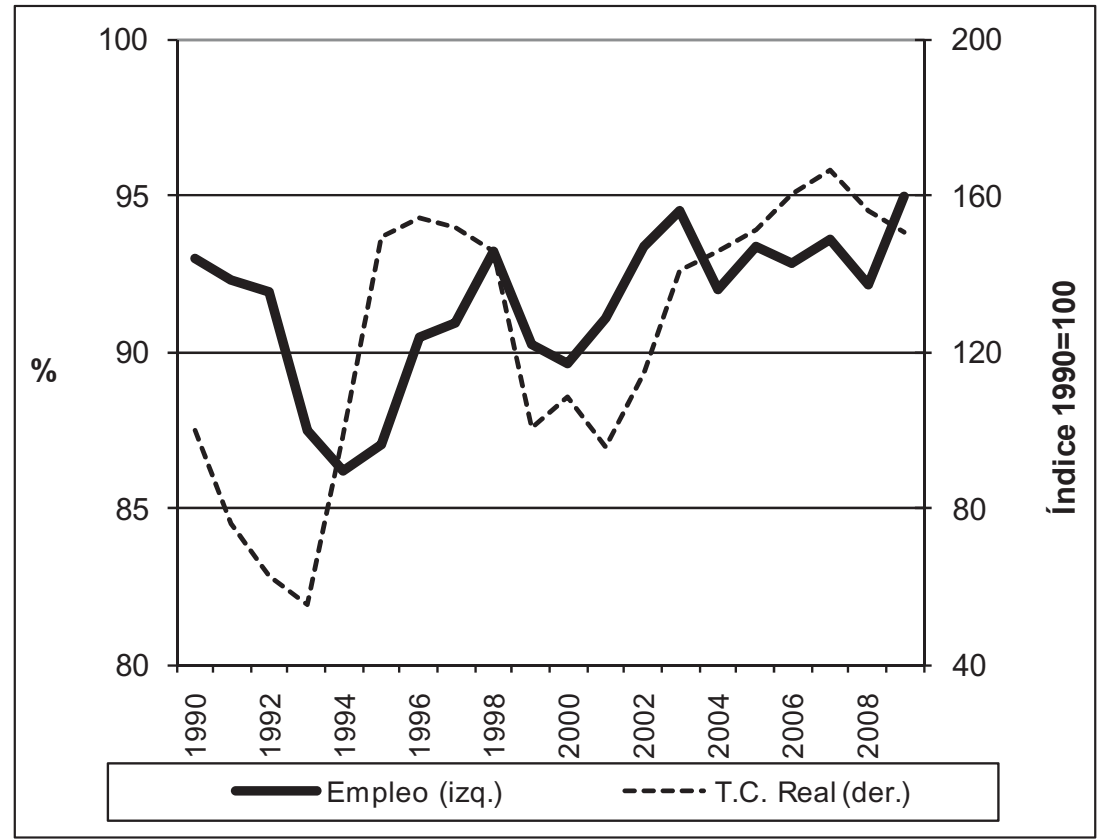

\footnotetext{
${ }^{1}$ Las tasas de empleo por departamento fueron tomadas del Instituto Nacional de Estadística (INE) y el tipo de cambio real se elaboró con base en cifras oficiales del Índice de Precios al Consumo y el tipo de cambio nominal de ambos países.
} 
La figura 2 muestra que el tipo de cambio real experimentó fuertes fluctuaciones en el periodo considerado. A su vez, se observa que las mismas han repercutido en la evolución del empleo en los departamentos fronterizos, apartándolo del comportamiento en el resto del país.

A modo de ejemplo basta mencionar cuatro momentos donde se observa la mencionada asociación entre las variables. En primer lugar, el deterioro experimentado por el tipo de cambio real bilateral desde el inicio del periodo hasta 1993 determinó que el empleo en la frontera exhibiera una tendencia negativa; en segundo lugar, la gran recuperación del mismo en el bienio 1994-1995 se tradujo en una mejora de la ocupación; en tercer lugar, la fuerte devaluación de la moneda brasileña ocurrida en 1999 quebró la tendencia anterior y determinó una nueva reducción del empleo; y finalmente, la devaluación uruguaya de 2002, cuyos efectos se prolongaron hasta el año siguiente, nuevamente produjo una gran mejora de la ocupación.

Si bien la gráfica muestra que existe la asociación prevista entre las variables, la misma se encuentra relativizada por dos aspectos. En primer lugar, cabe señalar que las tasas de empleo a nivel de departamentos son bastante volátiles, y los departamentos fronterizos no son la excepción en este sentido. Esto es resultado de que los tamaños de las muestras con que se calculan dichas tasas son relativamente pequeños.

En segundo lugar, parece haber un rezago entre las variaciones del tipo de cambio real y su impacto sobre el empleo. Esto se puede visualizar claramente en aquellos años, tales como 1994 y 2000, en que, si bien existe una mejora de la competitividad relativa de Uruguay, el empleo se sigue contrayendo seguramente como resultado del fuerte deterioro experimentado por la misma en los dos periodos previos.

Este fenómeno tiene sustento en la teoría económica. Como se sabe, el empleo es una variable que demora en ajustarse, en la medida en que existen costos vinculados tanto al despido (compensaciones monetarias) como a la incorporación de trabajadores (formación y capacitación). Teniendo en cuenta dichos costos, las empresas prefieren en el corto plazo ajustar las horas trabajadas para posteriormente, en el mediano plazo, de confirmarse las tendencias, realizar cambios en la plantilla.

La constatación de la existencia del mencionado rezago implicó que el mismo fuera tenido en cuenta en las estimaciones econométricas. Para 
esto, se pusieron a prueba diferentes alternativas en busca del mejor ajuste entre las variables.

\section{Estimaciones econométricas}

A los efectos de comprobar si el modelo desarrollado anteriormente recoge de manera adecuada los principales determinantes de la evolución del empleo en los departamentos fronterizos -Artigas y Rivera-, se realizaron estimaciones econométricas por mínimos cuadrados ordinarios utilizando datos semestrales correspondientes al periodo 1990-2009, sobre un total de 40 observaciones para cada departamento.

La variable dependiente en las dos ecuaciones estimadas es la tasa de empleo $^{2}$ en el departamento fronterizo: Artigas (TEA) y Rivera (TER). A su vez, las variables explicativas son la tasa de empleo en Montevideo (TEM) y el tipo de cambio real bilateral entre Uruguay y Brasil (TCRB).

$$
\begin{gathered}
T E A_{t}=\alpha+\beta * T E M_{t}+\delta * T C R B_{t-1}+\varepsilon_{t} \\
T E R_{t}=\theta+\sigma * T E M_{t}+\lambda * T C R B_{t-1}+\omega_{t}
\end{gathered}
$$

La primera variable explicativa (TEM) recoge el efecto que tiene la evolución del nivel de actividad económica en el conjunto del país sobre el empleo en todos los departamentos. Por tanto, el signo esperado de sus coeficientes es positivo.

La segunda variable explicativa (TCRB) recoge el efecto que tiene la evolución de los precios relativos entre Brasil y Uruguay sobre el empleo, efecto que es específico de los departamentos fronterizos. También aquí el signo esperado de los coeficientes es positivo. Corresponde aclarar que esta variable se tomó con el rezago de un periodo o semestre porque así presenta una mayor significación.

En primer lugar, se realizó la estimación en niveles poniendo a prueba la existencia de una relación de equilibrio en el largo plazo (cointe-

\footnotetext{
${ }^{2}$ En la medida en que el INE publica únicamente datos anuales de la tasa de empleo por departamento, fue necesario elaborar series semestrales con base en los microdatos.
} 
gración) entre las variables, considerando que las tres son integradas de orden uno (1).

Las estimaciones confirmaron en el caso de los dos departamentos la existencia de una relación de cointegración entre las variables en la medida en que los residuos del modelo resultaron integrados de orden cero $\mathrm{I}(0){ }^{3}$ Asimismo, los parámetros estimados resultaron todos significativos y con los signos esperados (cuadro 1).

El ajuste de estos dos modelos medido por sus R2 es moderado $(0.50 \mathrm{y}$ 0.57). Esto se debe a la gran variabilidad que exhiben las tasas de empleo en los departamentos del interior del país.

\section{Cuadro 1}

Resultados de las estimaciones en niveles

\begin{tabular}{|c|c|c|c|c|}
\hline \multirow{2}{*}{ Variable dependiente } & \multicolumn{3}{|c|}{ Variables explicativas } & \multirow{2}{*}{ R2 } \\
\cline { 2 - 4 } & C & TEM & TCRB(-1) & \\
\hline TEA & $\begin{array}{c}8,21 \\
\text { (Artigas) }\end{array}$ & $\begin{array}{c}0,68^{* * *} \\
(0,90)\end{array}$ & $\begin{array}{c}0,04^{* * *} \\
(3,91)\end{array}$ & \multirow{2}{*}{0,50} \\
\hline TER & $\begin{array}{c}3,42 \\
\text { (Rivera) }\end{array}$ & $\begin{array}{c}0,81^{* * *} \\
(0,47)\end{array}$ & $\begin{array}{c}0,02^{*} \\
(1,80)\end{array}$ & \multirow{2}{*}{0,57} \\
\hline
\end{tabular}

Los valores entre paréntesis corresponden a los estadísticos t. Los asteriscos indican si los parámetros son significativamente distintos de cero al: $90 \%\left(^{*}\right), 95 \%\left(^{* *}\right)$ y $99 \%(* * *)$ de confianza.

Fuente: Elaboración propia.

Posteriormente, a fin de estudiar la dinámica de corto plazo existente entre las variables se estimaron los modelos de corrección de error. Para esto se realizó la regresión en primeras diferencias de las mismas variables anteriormente consideradas: la tasa de empleo en el departamento fronterizo, ya sea Artigas ( $\Delta$ TEA) o Rivera $(\Delta$ TER); la tasa de empleo en Montevideo $(\Delta \mathrm{TEM})$; y el tipo de cambio real bilateral $(\Delta$ TсRв). Como variable explicativa se agregó el residuo de la regresión anteriormente estimada en niveles (RES), tomado con un periodo de rezago.

${ }^{3}$ Adicionalmente, la existencia de una relación de cointegración fue confirmada por el test de Johansen. 
Esta última variable recoge el efecto de cómo incide el desajuste que existe entre las variables, de acuerdo con la relación de equilibrio de largo plazo, en la dinámica de corto plazo. El signo esperado de su coeficiente es negativo en la medida en que la variable dependiente debería disminuir si en el periodo anterior el valor real de la misma se encontraba por encima de su equilibrio de largo plazo, y viceversa.

Los resultados de las estimaciones muestran que el ajuste de los modelos es superior al caso anterior $(0.61$ y 0.51$)$ y el signo de las variables es el esperado (cuadro 2). No obstante, la única variable significativa en ambos casos fue la correspondiente al desajuste en la relación de largo plazo. Además resultó significativa la tasa de empleo de Montevideo solamente en la estimación correspondiente al departamento de Rivera.

\section{Cuadro 2}

Resultados de las estimaciones de los modelos de corrección del error

\begin{tabular}{|c|c|c|c|c|c|}
\hline \multirow[t]{2}{*}{ Variable dependiente } & \multicolumn{4}{|c|}{ Variables explicativas } & \multirow[t]{2}{*}{$\mathbf{R 2}$} \\
\hline & C & $\Delta$ TEM & $\Delta \mathrm{TCRB}(-1)$ & $\operatorname{RES}(-1)$ & \\
\hline $\begin{array}{c}\Delta \text { TEA } \\
\text { (Artigas) }\end{array}$ & $\begin{array}{c}0,14 \\
(0,35)\end{array}$ & $\begin{array}{c}0,38 \\
(1,16)\end{array}$ & $\begin{array}{c}0,01 \\
(0,56)\end{array}$ & $\begin{array}{c}-1,17^{\star \star \star} \\
(-7,24)\end{array}$ & 0,61 \\
\hline $\begin{array}{c}\Delta \text { TER } \\
\text { (Rivera) }\end{array}$ & $\begin{array}{c}-0,06 \\
(-0,18)\end{array}$ & $\begin{array}{c}0,81^{\text {*** }} \\
(3,12)\end{array}$ & $\begin{array}{c}0,01 \\
(0,73)\end{array}$ & $\begin{array}{c}-0,84^{* * *} \\
(-5,09)\end{array}$ & 0,51 \\
\hline
\end{tabular}

Los valores entre paréntesis corresponden a los estadísticos t. Los asteriscos indican si los parámetros son significativamente distintos de cero al: $90 \%\left(^{*}\right), 95 \%(* *)$ y $99 \%(* * *)$ de confianza.

Fuente: Elaboración propia.

Esto pone de manifiesto que la dinámica de corto plazo de las variables dependientes está básicamente determinada por el ajuste necesario para alcanzar el equilibrio de largo plazo. Como confirmación de esto, cabe señalar que los coeficientes estimados de esta variable no son significativamente distintos de uno en ambos casos (-1.17 y -0.84), lo que indica que el ajuste mencionado se produce por completo en un solo semestre.

\section{Conclusiones}

El fenómeno del consumo transfronterizo de bienes es bien conocido en Uruguay. No obstante, hasta el momento no existían estimaciones del im- 
pacto que el mismo tiene sobre el empleo. En el presente trabajo se presentan las primeras cuantificaciones de la sensibilidad que tiene la tasa de empleo en los departamentos fronterizos respecto a las fluctuaciones del tipo de cambio real bilateral entre Brasil y Uruguay.

Las estimaciones econométricas confirman que el modelo presentado en este trabajo recoge adecuadamente los principales determinantes del comportamiento del empleo en los departamentos fronterizos. En particular, la tasa de empleo en Artigas y Rivera se correlaciona con la tasa del empleo en el resto del país. No obstante, además su evolución está determinada por otra variable: el tipo de cambio real bilateral con Brasil. Concretamente, el empleo mejora cuando los bienes uruguayos se abaratan en términos relativos y empeora cuando se encarecen comparativamente. En síntesis, el empleo en los dos departamentos fronterizos evidencia apartamientos de la tendencia del empleo a nivel nacional y dicho alejamiento se explica por las variaciones del tipo de cambio real bilateral.

Esto comprueba simultáneamente la significación que tienen dos fenómenos. En primer lugar, que en los departamentos analizados el consumo transfronterizo de bienes es un fenómeno altamente extendido entre la población y, como consecuencia de esto, es muy importante la porción de la demanda de bienes y servicios que cambia su lugar de compra de acuerdo con los precios relativos vigentes entre Brasil y Uruguay.

En segundo lugar, las estimaciones comprueban que estas fluctuaciones de la demanda entre los dos países se traducen en variaciones en la tasa de empleo en la margen uruguaya. Esto último, por su parte, se produce porque no existe un mercado laboral único entre las ciudades vecinas, sino más bien existen mercados segmentados con barreras a la entrada de los trabajadores de la margen vecina. De lo contrario, las fluctuaciones en la generación de empleo a cada lado de la frontera no afectaría la tasa de empleo de la población que habita en cada margen, pues la misma trabajaría indistintamente en ambos países.

En resumen, es posible afirmar que los mercados de trabajo no se encuentran lo suficientemente integrados entre las ciudades vecinas de ambas márgenes de la frontera como para amortiguar los efectos derivados de las fluctuaciones del consumo transfronterizo.

Por esto, corresponde señalar que el fenómeno del consumo transfronterizo, así como tiene sus ventajas en términos de un menor costo de 
vida para la población, también tiene su desventaja en la medida en que introduce mayor volatilidad en el empleo.

Ante esta realidad, y a fin de disminuir la volatilidad del empleo, es posible reaccionar con dos enfoques de política. El primero consiste en cerrar la frontera y evitar que la demanda de bienes se fugue hacia el país vecino y el segundo supone integrar más los dos mercados laborales hoy segmentados.

El primer enfoque mencionado, más allá de las dificultades de su implementación, sobre todo teniendo en cuenta la proximidad de las ciudades vecinas, implicaría perder la ventaja que el consumo transfronterizo supone en términos de costo de vida para la población. Por el contrario, el segundo enfoque no resigna este aspecto positivo, aunque supone mejorar los canales de información entre países sobre las oportunidades de empleo, así como facilitar o eliminar los trámites formales para trabajar al otro lado de la frontera.

En síntesis, la implementación de todas aquellas medidas de política que faciliten la libre movilidad del factor trabajo entre las ciudades vecinas de Brasil y Uruguay tenderá a reducir las fluctuaciones de la tasa de empleo en ambas poblaciones, sin privar a las mismas del beneficio que implica el consumo transfronterizo en términos de un menor costo de vida.

\section{Bibliografía}

Campbell, Jeffrey y Beverly Lapham (2004), "Real Exchange Fluctuations and the Dynamics of Retail Trade Industries on the U.S. - Canadá Border", The American Economic Review, 94, 4:1194-1206.

Correa, Fernando, (2006), "El efecto sustitución y la evolución del costo de vida en la frontera de Uruguay con Brasil”, Estudios Fronterizos, Nueva época, 7, 14:33-54.

Enders, Walter (1995), Applied Econometric Time Series, Jhon Wiley \& Sons, Nueva York.

Engel, Charles y John Rogers (1996), "How Wide is the Border", The American Economic Review, 86, 5:1112-1125.

Fullerton, Thomas (2000), "Currency Movements and International Border Crossings", International Journal of Public Administration, 23:1113-1123.

Harrell, Louis y Dale Fischer (1989), "The Mexican Peso Devaluation and Border Area Employment”, Southwest Journal of Business E Economics, 6:19-27.

INE (2006). Metodología de la Encuesta Nacional de Hogares ampliada, Montevideo. 
INE (2001), Metodología de la Encuesta Continua de Hogares, Montevideo.

McConnell, Campbell y Stanley Brue (1997), Economía laboral contemporánea. McGraw Hill, Madrid.

Parley, David y Shang Jin Wei (2001), "Explaining the Border Effect: The Role of Exchange Variability, Shipping Costs, and Geography", Journal of International Economics, 55, 1: 87-105.

Varian, Hal, (1992), Análisis macroeconómico, Antoni Bosch, Barcelona.

Wooldridge, Jeffrey (2001), Introducción a la econometría. Un enfoque moderno, Internacional Thomson Editores, México. 
